\title{
Potential Alzheimer's Disease Therapeutics Among Weak Cysteine Protease Inhibitors Exhibit Mechanistic Differences Regarding Extent of Cathepsin B Up-Regulation and Ability to Block Calpain
}

\author{
Heather Romine, $M B A$ \\ Katherine M. Rentschler \\ Kaitlan Smith \\ Ayanna Edwards \\ Camille Colvin \\ Karen Farizatto, PhD \\ Morgan C. Pait
}

University of North Carolina - Pembroke, Pembroke, North Carolina, USA

David Butler, PhD

Center for Drug Discovery, Northeastern University,

Boston, Massachusetts, USA

Ben A. Bahr, PhD

William C. Friday Laboratory,

University of North Carolina - Pembroke, North Carolina, USA

Doi: 10.19044/esj.2017.c1p5 URL:http://dx.doi.org/10.19044/esj.2017.c1p5

\begin{abstract}
Cysteine protease inhibitors have long been part of drug discovery programs for Alzheimer's disease (AD), traumatic brain injury (TBI), and other disorders. Select inhibitors reduce accumulating proteins and AD pathology in mouse models. One such compound, Z-Phe-Aladiazomethylketone (PADK), exhibits a very weak $\mathrm{IC}_{50}(9-11 \mu \mathrm{M})$ towards cathepsin B (CatB), but curiously PADK causes marked up-regulation of the $\mathrm{A} \beta$-degrading $\mathrm{CatB}$ and improves spatial memory. Potential therapeutic and weak inhibitor E64d (14 $\left.\mu \mathrm{M} \mathrm{IC}_{50}\right)$ also up-regulates CatB. PADK and E64d were compared regarding the blockage of calcium-induced cytoskeletal deterioration in brain samples, monitoring the $150-\mathrm{kDa}$ spectrin breakdown product (SBDP) known to be produced by calpain. PADK had little to no effect on SBDP production at 10-100 $\mu \mathrm{M}$. In contrast, E64d caused a dosedependent decline in SBDP levels with an $\mathrm{IC}_{50}$ of 3-6 $\mu \mathrm{M}$, closely matching
\end{abstract}


its reported potency for inhibiting $\mu$-calpain. Calpain also cleaves the cytoskeletal organizing protein gephyrin, producing $49-\mathrm{kDa}$ (GnBDP49) and 18-kDa (GnBDP18) breakdown products. PADK had no apparent effect on calcium-induced gephyrin fragments whereas E64d blocked their production. E64d also protected the parent gephyrin in correspondence with reduced BDP levels. The findings of this study indicate that PADK's positive and selective effects on CatB are consistent with human studies showing exercise elevates $\mathrm{CatB}$ and such elevation correlates with improved memory. On the other hand, E64d exhibits both marginal CatB enhancement and potent calpain inhibition. This dual effect may be beneficial for treating AD. Alternatively, the potent action on calpain-related pathology may explain E64d's protection in $\mathrm{AD}$ and TBI models.

Keywords: Alzheimer's disease, calpain, lysosome, PADK, E64d

\section{Introduction}

The age-related protein accumulation disorder Alzheimer's disease (AD) afflicts more than 5 million people in the U.S., also causing stress to caregivers, family, and friends. Furthermore, the disease greatly burdens a healthcare system that is incurring over $\$ 250$ billion in costs each year. The process of aging is known to exacerbate pathogenic protein accumulation, as most non-familial cases of AD occur in people 65 years or older. Features of AD include $i$ ) intracellular aggregates, ii) extracellular deposits, and iii) multiproteinopathic components comprising of $A \beta_{42}$, amyloid precursor protein (APP) fragments, hyperphosphorylated tau, and often TDP-43 and $\alpha$ synuclein (Nixon, 2007; Mazzuli et al., 2016). Protein accumulations occur prior to the onset of synaptic pathology and neurodegeneration (Bendiske et al., 2002; Goldberg, 2003; Butler et al., 2005), likely as a result of an imbalance between protein synthesis and degradation.

Lysosomes contain a variety of cysteine, aspartyl, and serine proteases as well as other enzymes as part of a key protein degradation pathway. Cathepsins are among the proteolytic enzymes located within the acidic environment of the lysosome where their main function is the degradation of large biomolecules (see Bahr, 2009; Ditaranto et al., 2001; Wang et al., 2017). The specific enzyme cathepsin B (CatB) has been implicated as a therapeutic target for $\mathrm{AD}$ since it degrades $\mathrm{A} \beta_{42}$ into less pathogenic peptides through $\mathrm{C}$ terminal truncation (Mueller-Steiner et al., 2006; Butler et al., 2011). In addition, positive $\mathrm{CatB}$ modulation appears to be a cellular protection avenue since the enzyme exhibits an enhancement effect in response to many types of protein clearance compromise and protein accumulations, including:

- Proteasome inhibitor treatment in SH-SY5Y cells (Cecarini et al., 2014) 
- Proteasome inhibition in SH-SY5Y cells expressing human APP (Cecarini et al., 2014)

- Intrahippocampal injection of proteasome inhibitor in aged rats (Gavilán et al., 2015)

- Proteasome inhibitor treatment in hippocampal slices (Farizatto et al., 2017)

- $\quad$ Chloroquine-induced lysosomal stress in hippocampus (Bendiske \& Bahr, 2003).

- High concentration $A \beta_{42}$ in hippocampal slices (Bendiske \& Bahr, 2003)

- $\quad$ Low-levels of $A \beta_{42}$ in hippocampal slices (Farizatto et al., 2017)

- $\mathrm{A} \beta_{42}$ treatment of a mouse neuronal cell line (Mueller-Steiner et al., 2006)

- $\quad$ Expression of mutant human APP in mouse brain (Mueller-Steiner et al., 2006)

- $\quad$ Human huntingtin expression in cultured neurons (Wu et al., 2012)

Note that a compensatory inter-relationship between the proteasomal and lysosomal protein clearance pathways has been suggested with recent evidence showing that impairment of the proteasomal system activates CatB and the lysosomal system (Cecarini et al., 2012; Farizatto et al., 2017). In addition, a positive modulator of the lysosomal system enhanced the level of CatB activity and also reduced $A \beta$ accumulation in neuroblastoma cells expressing mutant APP (Park et al., 2016). Across AD transgenic mouse models, genetic and pharmacological manipulations found to increase CatB activity also reduced $A \beta$ levels and offset disease parameters (Mueller-Steiner et al., 2006; Sun et al., 2008; Butler et al., 2011; Yang et al., 2011; Wang et al., 2012).

In other studies, $\mathrm{CatB}$ inhibition has been linked to the reduction of $\mathrm{A} \beta$ deposits and improved memory in $\mathrm{AD}$ mouse models using the broad cysteine protease inhibitor E64d (see Hook et al., 2007, 2011, 2014a). E64d, however, was also found to potently inhibit the calcium-activated protease calpain and such inhibition was found to protect against different neuropathologies (Inubushi et al., 1994; Tsubokawa et al., 2006; Trinchese et al., 2008; Jeon et al., 2016). Thus, the current study compared the distinct compounds PADK and E64d with regard to the extent of their CatB modulation vs. their calpain blocking capacity. The latter was assessed by measuring calcium-dependent breakdown of the cytoskeletal protein spectrin that is implicated in many pathological states (Vanderklish \& Bahr, 2000; Pineda et al., 2004; Weiss et al., 2009; Ono et al., 2016) and the calcium-dependent breakdown of gephyrin, a postsynaptic scaffold protein that recruits transmitter receptors and interacts 
with a guanine nucleotide exchange factor (Kawasaki et al., 1997; Fekete et al., 2017).

\section{Materials and Methods}

Hippocampal Slice Cultures. All studies with animals were carried out in accordance with the recommendations from the Guide for the Care and Use of Laboratory Animals from the National Institutes of Health. Brain tissue from postnatal 12-day-old Sprague-Dawley rats (Charles River Laboratories) was rapidly removed to prepare hippocampal slices (Farizatto et al., 2017; Butler et al., 2011). Transverse slices $(400 \mu \mathrm{m})$ were quickly prepared and gently positioned on Millicell-CM inserts (Millipore, Billerica, Massachusetts). The hippocampal slices were maintained at $37^{\circ} \mathrm{C}$ in $5 \% \mathrm{CO}_{2}-$ enriched atmosphere for 18-22 days before being treated with different agents.

Culture Treatments. E64d (2S,3S-trans-epoxysuccinyl-Lleucylamido-3-methylbutane ethyl ester or aloxistatin; Sigma-Aldrich; St. Louis, Missouri) was applied daily to hippocampal slice cultures for 2-3 days. The compound Z-Phe-Ala-diazomethylketone (PADK; Bachem Inc., Torrance, California), which promotes mature CatB levels in different model systems (Ryzhikov \& Bahr, 2008; Butler et al., 2011; Bahr et al., 2012) was similarly assessed in the hippocampal cultures. After treatments, cultured slices were gently removed from the inserts into groups of 7-9 each using icecold isosmotic buffer and homogenates were prepared.

Cathepsin B Activity. The InnoZyme Assay Kit (Millipore) was used to measure CatB activity in the hippocampal slice samples treated with various concentrations of compounds. Aliquots of homogenized samples (10 $\mu \mathrm{g}$ protein) were assessed in duplicate for proteolytic activity using the Z-ArgArg AMC substrate and the SpectraMax M3 microplate reader.

Calcium-Dependent Calpain Assay. Brain tissue homogenates were rapidly prepared from adult rats, which were humanely sacrificed by isoflurane anesthesia and decapitation. Brains were cooled and removed from the skull and then placed in ice-cold homogenization buffer. The brains were immediately dissected and telencephalic tissue was collected, homogenized and assayed for protein content level. Equal protein aliquots were incubated with $6 \mathrm{mM} \mathrm{CaCl}_{2}$ at $37^{\circ} \mathrm{C}$, in the absence or presence of potential protease inhibitors, and then assessed by immunoblot for proteolytic products.

Immunoblot Analysis. Immunoblot samples of adult brain tissue and hippocampal slice cultures were sonicated in cold lysis buffer (Sigma-Aldrich; St. Louis, Missouri). Protein content was determined and equal amounts of protein were denatured in sample buffer and separated on gradient gels for subsequent transfer to nitrocellulose. Blots were incubated in blocking solution containing 5\% milk or BSA for $1 \mathrm{~h}$. Primary antibody staining utilized antibodies against cathepsin B (1:100, Calbiochem), GluR1 (1:1000; 
Millipore) and anti- $\alpha$ II spectrin (1:100, Santa Cruz), as well as against actin 20-33 (1:500, Sigma) and an antibody to gephyrin's C-terminal (1:250) made against the sequence VELHKGEVVDVMVIGRL described in Kawasaki et al. (1997). Anti-IgG-alkaline phosphatase conjugates and anti-IgGhorseradish peroxidase conjugates were used for the secondary antibody step, and antigen staining and image development involved the chemiluminescence protocols using the GE Amersham AI600RGB imager. Immunostained bands were scanned at high resolution to determine integrated optical density with BIOQUANT software (R \& M Biometrics, Nashville, Tennessee).

Transgenic Mice Assessment. Transgenic and control mice were housed in vivarium facilities until the desired age. The $\mathrm{APP}_{\text {SwInd }} \mathrm{J} 20$ line mice (Jackson Laboratories) exhibit lower levels of $A \beta$ deposits compared to the original line, and were used at 9-10 months of age. Genotype was confirmed by PCR. Mice were handled daily for 1 week and subsequently received daily i.p. injections of $20 \mathrm{mg} / \mathrm{kg}$ PADK. Control mice were injected with the corresponding volume of vehicle. Mice were handled and familiarized with the open field used for spatial memory. The $\mathrm{APP}_{\text {SwInd }}$ mice and age-matched wild-type mice were mildly caloric-restricted and assessed for spatial memory in the hidden food cache test. A food reward was placed in one of two opaque cylinders in opposite corners of an open field. The food location was changed after each 3-trial training session over a 24 -h period, and food retrieval time was compared across trials.

Statistical Analyses. Specific immunoreactivity values [(optical density - background) $\times$ area] for each antigen were quantitatively compared. Results were evaluated with unpaired $t$ tests or analyses of variance (ANOVA) followed by post hoc tests using Prism software (GraphPad, San Diego, California). $\mathrm{IC}_{50}$ and $\mathrm{EC}_{50}$ values were determined using nonlinear regression.

\section{Results}

Potential targets of the weak cysteine protease inhibitors PADK and E64d include the lysosomal system and the calcium-activated protease calpain (Fig. 1a). PADK was assessed for CatB inhibitory action in brain homogenates, resulting in a very weak $\mathrm{IC}_{50}$ value of 9-10 $\mu \mathrm{M}$ (Fig. 1b). In contrast, lower PADK concentrations elicited positive modulation of the 30$\mathrm{kDa}$ CatB active isoform (CatB-30) in hippocampal slice cultures (Fig. 1c), including concentrations that produced no or minimal inhibitory effects on the enzyme (see grey zones in Figs. 1b and 1c). 

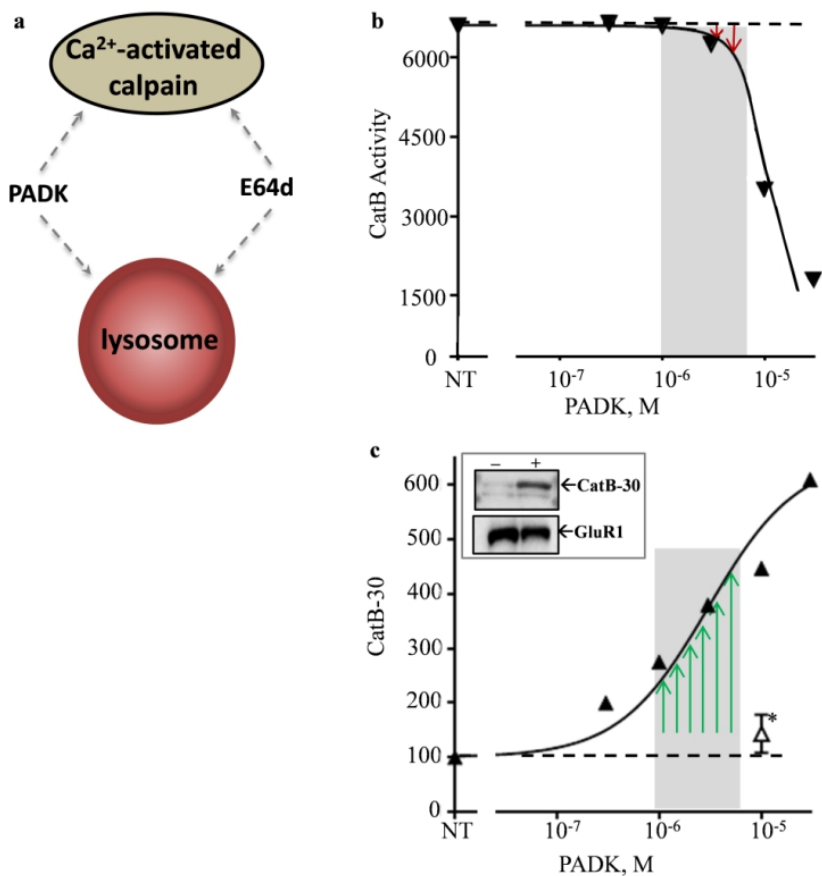

Figure 1. Potential enzyme modulation avenues. PADK and E64d are protease inhibitors that potentially target calpain and/or lysosomal hydrolases (a). PADK was assessed for its effect on CatB activity (b), exhibiting an $\mathrm{IC}_{50}$ of $9-10 \mu \mathrm{M}$ in brain homogenates. PADK at 1-8 $\mu \mathrm{M}$ (grey zone) produced little inhibition (red arrows), while the same concentrations enhanced the CatB-30 active isoform 3-5 fold (c; green arrows) in hippocampal slice cultures $\left(\mathrm{EC}_{50}=2.8 \mu \mathrm{M}\right)$. GluR1 was unchanged in PADK-treated samples $(+)$. In the presence of the CatB inhibitor CA074, $10 \mu \mathrm{M}$ PADK was unable to have an effect on CatB-30 $(* \mathrm{p}<0.05)$.

PADK's dose-dependent modulatory effect on CatB-30 in Figure 1c exhibited an $\mathrm{EC}_{50}$ of $2.8 \mu \mathrm{M}$, thus was $>3$-times more potent than its weak inhibitory potency to explain its enhancing effect on CatB activity previously reported (Butler et al., 2005, 2011; Farizatto et al., 2017). This modulator induced a 3-6-fold increase in CatB-30 as compared to levels found in untreated hippocampal slices, and its effect was blocked by the very potent CatB inhibitor CA074 (open triangle in Fig. 1c). Corresponding with this CatB-enhancing effect, PADK improved spatial memory in APP ${ }_{\text {SwInd }}$ transgenic mice using a novel hidden food cache paradigm (Table 1). APP ${ }_{\text {SwInd }}$ and age-matched wildtype mice were trained to find the location of food that was placed in one of two opaque cylinders positioned in different configurations for each test. Wildtype mice exhibited improved time to find 
the food reward after three trials $(\mathrm{p}<0.001)$, whereas $\mathrm{APP}_{\mathrm{Sw} \text { Ind }}$ mice showed no improvement after 3 trials (Table 1). PADK treatment at $20 \mathrm{mg} / \mathrm{kg} /$ day (i.p.) allowed the transgenic mice to improve their food location time $(\mathrm{p}=0.036)$, to a level comparable to the performance by wildtype controls. These results are similar to the previously correlated improvement of episodic memory in APPPS1 mice (Butler et al., 2011).

seconds to find food cache (mean \pm SEM):

$\frac{\text { trial }}{1}$
$3+$ veh
$3+$ PADK

WT mice
$\begin{gathered}11.0 \pm 2.5 \\ 6.30 \pm 1.2 * * \\ -\end{gathered}$

APP $_{\text {SwInd mice }}$

$12.3 \pm 2.8$

$13.1 \pm 2.6$

$6.16 \pm 2.7^{*}$

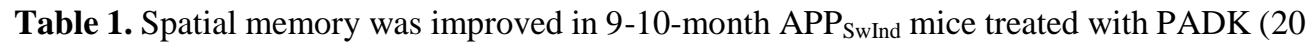
$\mathrm{mg} / \mathrm{kg} /$ day ip for 9 days). APP $_{\text {SwInd }}$ mice and age-matched wild-type mice (WT) were mildly caloric-restricted and assessed with the hidden food cache test. A food reward was placed in one of two opaque cylinders in opposite corners of an open field. The food location was changed after each 3-trial training session, and food retrieval time was compared across trials. $* * \mathrm{p}<0.001$, t-test compared to trial $1 ; * \mathrm{p}=0.036$, Mann-Whitney test compared to APP $\mathrm{SwInd}_{\text {. }}$ trial $3+$ vehicle.

The broad cysteine protease inhibitor E64d also has been implicated as a treatment avenue for AD (Hook et al., 2007, 2011, 2014a). While it has been suggested that E64d's inhibitory effect on CatB is the underlying therapeutic action for offsetting $\mathrm{AD}$ pathology, this weak protease inhibitor was found to increase CatB-30 (Table 2). Note that the positive modulation of CatB by E64d was less pronounced than the enhancing effect produced by PADK (increase of $76 \%$ vs. $549 \%$; $p<0.001$ ). Other weak cysteine protease inhibitors were also found to up-regulate active CatB to varying degrees, including $i$ ) SD1002, a non-peptidyl PADK analogue previously found to promote $\mathrm{A} \beta_{42}$ clearance (Viswanathan et al., 2012), ii) the polyphenol quercetin that, like PADK and E64d, exhibits very weak CatB inhibitory action (Ramalho et al., 2015), and iii) Cathepsin Inhibitor 1 (CATI-1, also known as Z-Phe-Gly-NHO-Bz), a broad inhibitor of papain and several cathepsins (Table 2). The potent CatB inhibitors CA074, CA074me, and E64 did not exhibit positive modulation of CatB-30. Table 2 also compared the listed 8 compounds for their inhibitory action on the calcium-activated cysteine protease calpain and, interestingly, E64d and the related E64 compound stand out as the most potent. 


\begin{tabular}{|c|c|c|c|c|}
\hline \multirow{2}{*}{\multicolumn{2}{|c|}{$\begin{array}{l}\text { very weak inhibitors } \\
\text { vehicle control }\end{array}$}} & CatB-30, percent \pm SEM & $\mathrm{IC}_{50}$ for CatB, $\mu \mathrm{M}$ & $\underline{\mathrm{IC}}_{50}$ for calpain, $\mu \mathrm{M}$ \\
\hline & & $100 \pm 4.0$ & - & - \\
\hline PADK & $\begin{array}{r}1 \mu \mathrm{M} \\
10 \mu \mathrm{M}\end{array}$ & $\begin{array}{l}340 \pm 38.7^{* *} \\
649 \pm 74.5^{* * *}\end{array}$ & $9-11^{\mathrm{a}}$ & $\geq 100$ (from Fig. 3d) \\
\hline E64d & $\begin{array}{r}1 \mu \mathrm{M} \\
10 \mu \mathrm{M}\end{array}$ & $\begin{array}{l}115 \pm 9.2 \\
176 \pm 12.5 * * * \# \#\end{array}$ & $14^{\mathrm{b}}$ & $\begin{array}{c}3-6 \text { (from Fig. 3b) } \\
0.04-4^{c}\end{array}$ \\
\hline SD1002 & $10 \mu \mathrm{M}$ & $345 \pm 13.3^{* *}$ & $>50^{\mathrm{d}}$ & n.d. \\
\hline quercetin & $10 \mu \mathrm{M}$ & $198 \pm 19.2 * *$ & $8^{e}$ & $211^{\mathrm{f}}$ \\
\hline CATI-1 & $10 \mu \mathrm{M}$ & $149 \pm 14.3^{* *}$ & weak inhibitor ${ }^{g}$ & $>20^{\mathrm{h}}$ \\
\hline potent is & bitors & CatB-30, percent \pm SEM & $\underline{\mathrm{IC}}_{50}$ for CatB, $\mu \mathrm{M}$ & $\underline{\mathrm{IC}}_{50}$ for calpain, $\mu \mathrm{M}$ \\
\hline CA074 & $0.3-1 \mu \mathrm{M}$ & $95 \pm 11.9$ & $0.004^{\mathrm{b}}$ & $>100^{\mathrm{b}}$ \\
\hline CA074me & $0.3-1 \mu \mathrm{M}$ & $116 \pm 9.2$ & $0.12^{\mathrm{a}}$ & n.d. \\
\hline E64 & $1-2 \mu \mathrm{M}$ & $112 \pm 7.5$ & $0.03^{\mathrm{i}}$ & $0.57^{\mathrm{j}}$ \\
\hline
\end{tabular}

Table 2. Comparisons among weak cysteine protease inhibitors and potent inhibitors regarding CatB-enhancing activity in hippocampal slice cultures and inhibitory activity targeting CatB and calpain. Measures of the 30-kDa CatB isoform (CatB-30) in treated hippocampal slices were from Farizatto et al. (2017). ANOVA multiple comparison tests compared to vehicle control: $* * \mathrm{p}<0.01, * * * \mathrm{p}<0.001$; unpaired t-test compared to $10 \mu \mathrm{M}$ PADK: ${ }^{\# \#} \mathrm{p}<0.001$. $\mathrm{IC}_{50}$ values for inhibiting calpain-mediated SBDP production are shown for PADK and E64d from Fig. 3. Other $\mathrm{IC}_{50}$ values for inhibiting CatB and calpain were obtained from the following references: a, Butler et al., 2011; b, Jeon et al., 2016; c, Huang et al., 1992; d, Viswanathan et al., 2012; e, Ramalho et al., 2015; f: Je Ma et al., 2009; g, 55; h, Montagne et al., 2017; i, Inubushi et al., 1994; j, Trinchese et al., 2008. CATI-1, Cathepsin Inhibitor 1; n.d., not determined.

To determine whether PADK and E64d have different actions on the calcium-activated calpain, assays were conducted to assess their ability to inhibit previously characterized breakdown products of spectrin and gephyrin mediated by calpain (SBDP and GnBDP; Fig. 2). Pathogenic calpain activation is known to cleave the $\alpha$-subunit of the cytoskeletal protein spectrin (Vanderklish \& Bahr, 2000). Calpain also cleaves the postsynaptic scaffold protein gephyrin into long-lasting fragments of 18 and $49 \mathrm{kDa}$ (Kawasaki et al., 1997). Brain homogenates were prepared and incubated with calcium, producing a $150-\mathrm{kDa}$ aII-spectrin fragment, and E64d inhibited this SBDP production (Fig. 3a). The dose-dependent inhibitory effect exhibited an $\mathrm{IC}_{50}$ of 3-6 $\mu \mathrm{M}$ (ANOVA $\mathrm{p}=0.0066$; Fig. 3b), closely matching E64d's reported potency for blocking $\mu$-calpain (Huang et al., 1992). PADK, on the other hand, did not decrease SBDP levels in calcium-treated brain samples (Fig. 3c). Insignificant variability in SBDP levels was found (Fig. 3d), but some calpain 
inhibition may occur at very high PADK concentrations that would negate its positive modulation of CatB.

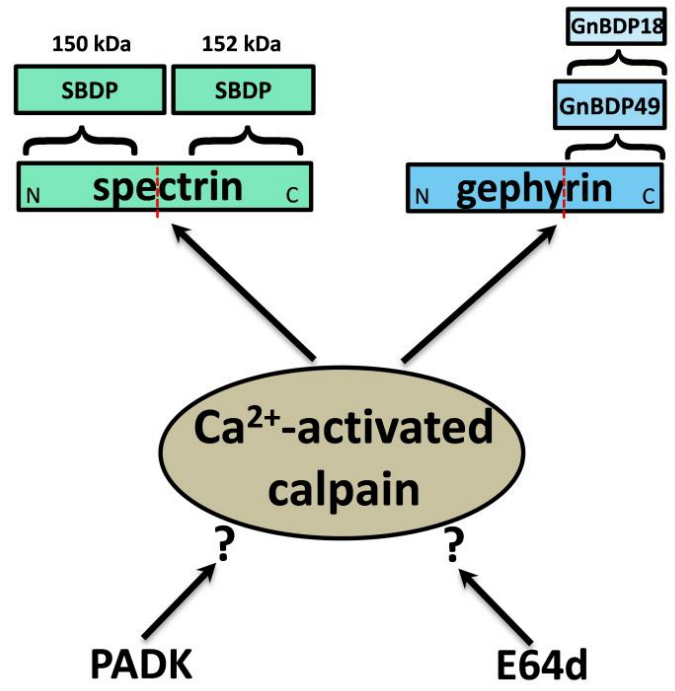

Figure 2. Calpain-mediated proteolysis of spectrin and gephyrin. Compounds may have an effect on calpain, a calcium-activated protease that generates breakdown products of spectrin (SBDPs of 150-152 kDa) and gephyrin (GnBDPs of 18 and $49 \mathrm{kDa}$ ).
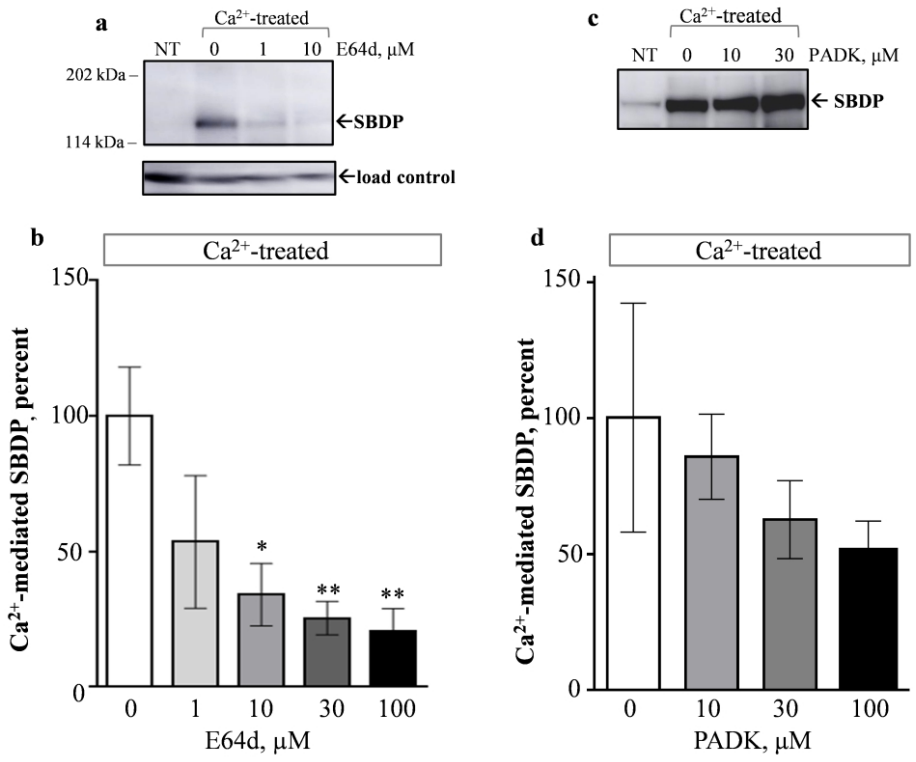

Fig. 3. E64d blocks calcium-induced spectrin breakdown while PADK does not. Rat telencephalic homogenates were not treated (NT) or treated with $\mathrm{CaCl}_{2}$ at $37^{\circ} \mathrm{C}$ for $1 \mathrm{~h}$ to activate proteases in the presence of E64d (a, b) or PADK (c, d). Equal protein aliquots were assessed by immunoblot for anti- $\alpha$ II-spectrin staining of the 150-kDa SBDP and for labeling of a gel loading control. Calcium-induced SBDP levels were normalized to the $0 \mu \mathrm{M}$ control and means \pm SEM were compared to the control: ${ }^{*} p<0.05,{ }^{* *} p \leq 0.01$. PADK had no effect on SBDP. 
Concerning the calpain-mediated gephyrin fragments previously characterized in hippocampal membranes, calcium treatment of rat telencephalic samples also led to the production of a $49-\mathrm{kDa}$ breakdown product (GnBDP49) as found in the hippocampal study, and to the production of a smaller 18-kDa fragment (GnBDP18) labeled by the antibodies developed against gephyrin's carboxyl-terminal sequence (Fig. 4a). Comparing the two distinct compounds of this study, PADK at $100 \mu \mathrm{M}$ was found to have no effect on the calcium-induced generation of GnBDP18 (Fig. 4b and 4c), whereas 100 $\mu \mathrm{M}$ E64d completely blocked the 18-kDa fragment from forming (Fig. 4c).

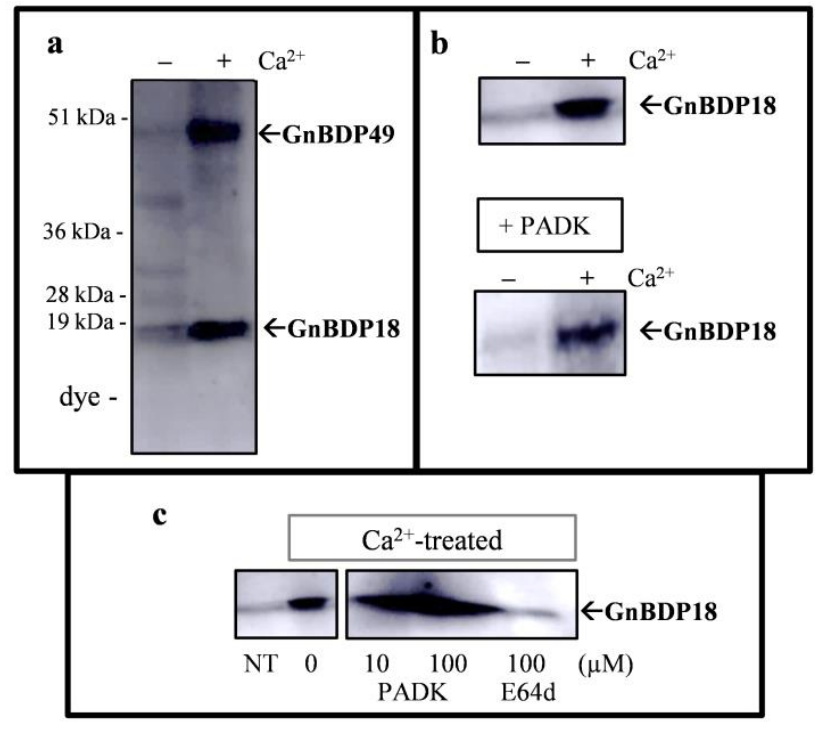

Figure 4. E64d blocks calcium-induced gephyrin breakdown. Telencephalic homogenates were treated without (-) or with $(+) \mathrm{CaCl}_{2}$ at $37^{\circ} \mathrm{C}$ for $1 \mathrm{~h}$, generating gephyrin breakdown products of 49 (GnBDP49) and $18 \mathrm{kDa}$ (GnBDP18) that were detected by immunoblot (a). Molecular weight markers are shown. PADK had no effect on calcium-induced GnBDP18 formation (b) whereas E64d blocked its formation (c). NT, not treated with $\mathrm{CaCl}_{2}$.

In addition to inhibiting the GnBDP18 proteolytic cleavage product, E64 blocked the formation of the larger GnBDP49 fragment as well (Fig. 5a and $5 \mathrm{~b}$ ). The dose-dependent reduction of the $49-\mathrm{kDa}$ cleavage product in calcium-activated samples closely corresponded with E64d's dose-dependent inhibition of spectrin breakdown (Fig. 5c). Note that the calcium-induced loss of the protein band shown as a gel load control was also protected by E64d. 
a

$\mathrm{Ca}^{2+}$-treated brain tissue

$\begin{array}{lllll}0 & 1 & 10 & 30 & \text { E64d, } \mu M\end{array}$

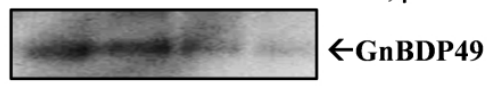

b

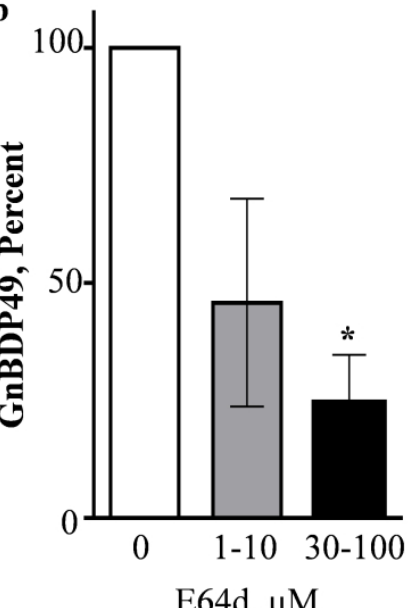

c
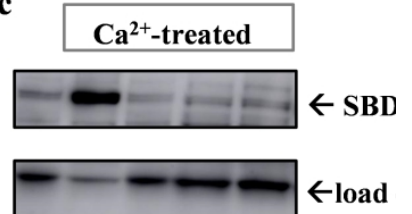

$\begin{array}{llllll}\text { NT } & 0 & 1 & 10 & 30 & \text { E64d, } \mu\end{array}$

Figure 5. E64d reduces GnBDP49 in calcium-treated brain tissue. Telencephalic homogenates were treated with $\mathrm{CaCl}_{2}$ at $37^{\circ} \mathrm{C}$ for $1 \mathrm{~h}$, generating the gephyrin fragment GnBDP49 detected by immunoblot (a) and the E64d inhibitory effect was determined across concentrations. Calcium-induced GnBDP49 levels were normalized to the $0 \mu \mathrm{M}$ control and means \pm SEM were compared to the control: ${ }^{*} p<0.0182$. A corresponding E64d effect was found for blocking calcium-induced SBDP levels (c). NT, not treated with $\mathrm{CaCl}_{2}$.

Next, we tested the E64d and PADK compounds for the ability to protect the parent gephyrin protein, the $95-\mathrm{kDa}$ isoform, from deterioration by calcium-induced proteolysis. In a pair of calcium-treated telencephalic homogenates, the brain sample with the addition of E64d exhibited a correspondence between blocking gephyrin fragment formation and protecting the parent gephyrin (see top two blot strips in Fig. 6a). As also indicated in the figure, the E64d inhibitor had similar abilities to 1) reduce gephyrin from being fragmented to GnBDP49 and other breakdown products and 2) reduce spectrin from being proteolyzed to the SBDP calpain cleavage product. 
$\mathbf{a}$

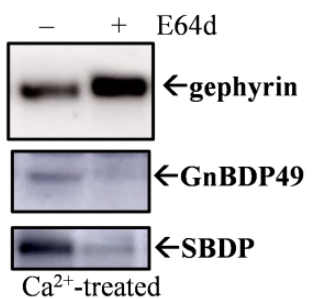

c

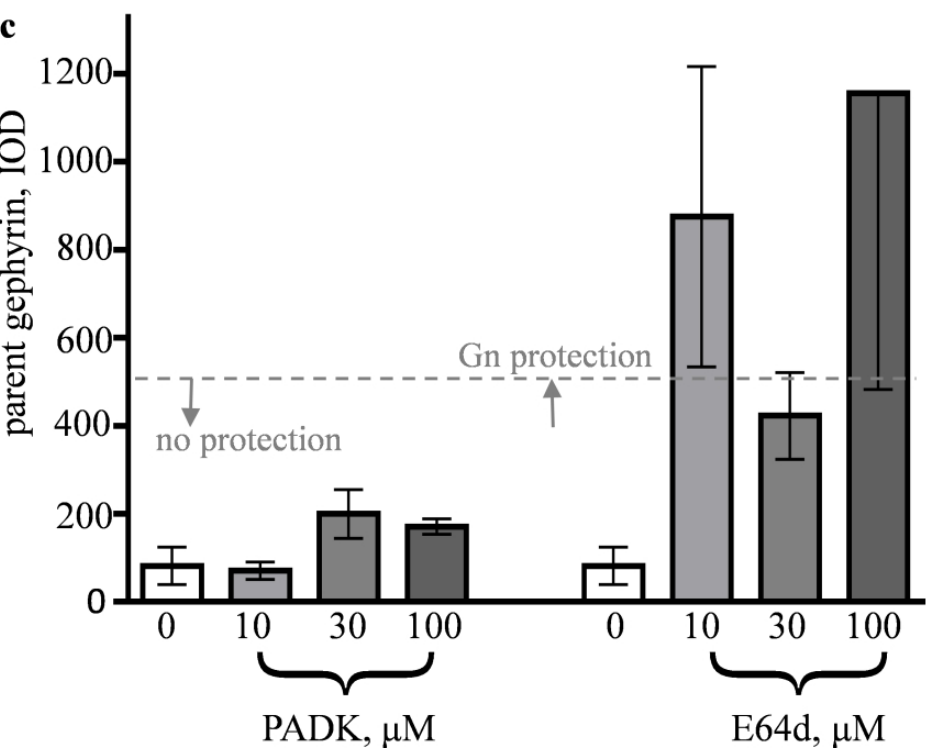

Figure 6. E64d protects the 95-kDa parent gephyrin from being proteolytically depreciated. The calcium-treated brain homogenates incubated in the absence $(-)$ or presence of E64d (+) were assessed by immunoblot for both the parent gephyrin and GnBDP49 as well as SBDP (a). PADK- and E64d-treated samples were assessed for protection of calcium-induced loss of gephyrin (b). Integrated optical densities (mean IODs \pm SEM) of gephyrin labeling were normalized to the $0 \mu \mathrm{M}$ control for PADK samples (no protection) and for E64d samples that exhibited protection to levels that were $\geq$ control levels (c). Dotted line: gephyrin amount in no-calcium control samples.

Additional calcium-treated brain samples were incubated with 10-100 $\mu \mathrm{M}$ PADK and showed no evidence that PADK protected the 95-kDa parent gephyrin, but samples treated with 10-100 $\mu \mathrm{M}$ E64d were associated with robust levels of protected gephyrin protein (Fig. 6b). When examining the effects across dosages of PADK and E64d, for evidence that they govern cytoskeletal decay, calcium-treated samples with different PADK concentrations fell well below the control gephyrin measures from samples without the calcium incubation (average control level noted by dotted line in Fig. 6c). This finding indicates that no protection of gephyrin was produced by PADK. In contrast, calcium-incubated samples with different E64d 
concentrations exhibited amounts of parent gephyrin that approached or exceeded the dotted-line control level of gephyrin (Fig. 6c). Interestingly, the calcium-activated homogenates that were treated with $10-100 \mu \mathrm{M} \mathrm{E} 64 \mathrm{~d}$ exhibited much more parent gephyrin than those homogenate samples that were treated with $100 \mu \mathrm{M}$ PADK. Together with results of the breakdown product experiments, these findings indicate that E64d protects gephyrin from calpain-mediated proteolytic damage.

\section{Discussion}

For over 30 years, research programs for developing neurotherapeutics and other disease treatments have included cysteine protease inhibitors (see Wang \& Yuen, 1994; Nixon, 2000; Vanderklish \& Bahr, 2000; Trinchese et al., 2008; Saatman et al., 2010; Ono et al., 2016; Sugiyama et al., 2017). For protein accumulation disorders (e.g., AD, Parkinson's disease, and Huntington's disease), it is counterintuitive to use protease inhibitors that would block the same protein clearance pathways whose dysfunctions are part of pathogenic cascades (see Torres et al., 2012; Burbulla et al., 2017; Farizatto et al., 2017). Pathogenic protein clearance is a critical issue for AD-type pathology since $A \beta_{42}$ and $A \beta_{40}$ peptides have impaired clearing rates in the human disease (Mawuenyega et al., 2010). Accordingly, a growing number of studies has investigated agents that enhance enzymes appropriately involved in protein clearance. The CatB cysteine protease is one such enzyme: it is a lysosomal hydrolase that degrades $\mathrm{A} \beta$ into less amyloidogenic species (Mueller-Steiner et al., 2006; Butler et al., 2011; Wang et al., 2012; Cermak et al., 2016; Park et al., 2016) and it is neuroprotective when up-regulated, reducing synaptic and behavioral deficits related to AD (Mueller-Steiner et al., 2006; Sun et al., 2008; Butler et al., 2011; Yang et al., 2011; Viswanathan et al., 2012). The PADK and E64d compounds compared here are both very weak inhibitors of the $A \beta$-degrading $C a t B$, but both were found to up-regulate $\mathrm{CatB}$ levels which may explain their beneficial actions in AD mouse models (see Butler et al., 2011; Hook et al., 2011; Bahr et al., 2012). It is noteworthy, however, that the two compounds exhibited distinct actions on the active CatB isoform vs. the calcium-dependent cysteine protease calpain:

- $\quad$ PADK up-regulated CatB-30 by 549\% while causing no apparent change in calpain activity;

- $\quad$ E64d up-regulated CatB-30 by $76 \%$ but reduced calpain activity by $80-90 \%$.

PADK has a $>7$-fold effect on CatB enhancement as compared to E64d's effect, as indicated by hippocampal slices after being treated with the compounds. PADK's robust effect on CatB explains the improved protein clearance as part of its protective actions in $\mathrm{AD}$ mouse models, which is consistent with human 
studies showing exercise elevates CatB levels in correlation with improved memory (Moon et al., 2016). E64d, on the other hand, elicits a much smaller enhancing effect on CatB, but this small enhancement may be part of E64d's therapeutic action at concentrations that do not block the protein clearing role of CatB.

This study also indicates that PADK and E64d differ mechanistically in their abilities to block calpain-mediated proteolysis. PADK's positive effect on CatB was found to be selective, apparently independent of any inhibitory effect on calpain, an enzyme linked to several neurodegenerative disorders including stroke, seizures, TBI, and AD (see reviews: Nixon, 2000; Vanderklish \& Bahr 2000). E64d's blocking of calpain-mediated cytoskeletal damage was observed to be many times more potent than its effect on CatB. Thus, PADK and E64d may provide separate yet promising therapeutic avenues by acting on different targets (see Fig. 7).

PADK increases CatB-30 levels through an interaction with the CatB enzyme's active site. Such an interaction appears to occur to different degrees by phenolic-rich structures including PADK, the related Z-Phe-Phediazomethylketone, the nonpeptidic modulator SD1002, and the natural phenolic compound quercetin since they were effective at up-regulating CatB30. In contract, the structurally distinct epoxysuccinyl peptide E64d acted 7fold less potently than PADK for CatB positive modulation. In addition to lacking phenolic motifs that may facilitate the modulation, E64d is known to be hydrolyzed once it readily permeates into cells, becoming a potent cysteine protease inhibitor with an $\mathrm{IC}_{50}$ for blocking calpain of $0.04 \mu \mathrm{M}$ (Huang et al., 1992). This feature of E64d likely influences its ability to up-regulate CatB since the better positive CatB modulators identified here are very weak inhibitors of cysteine proteases. 


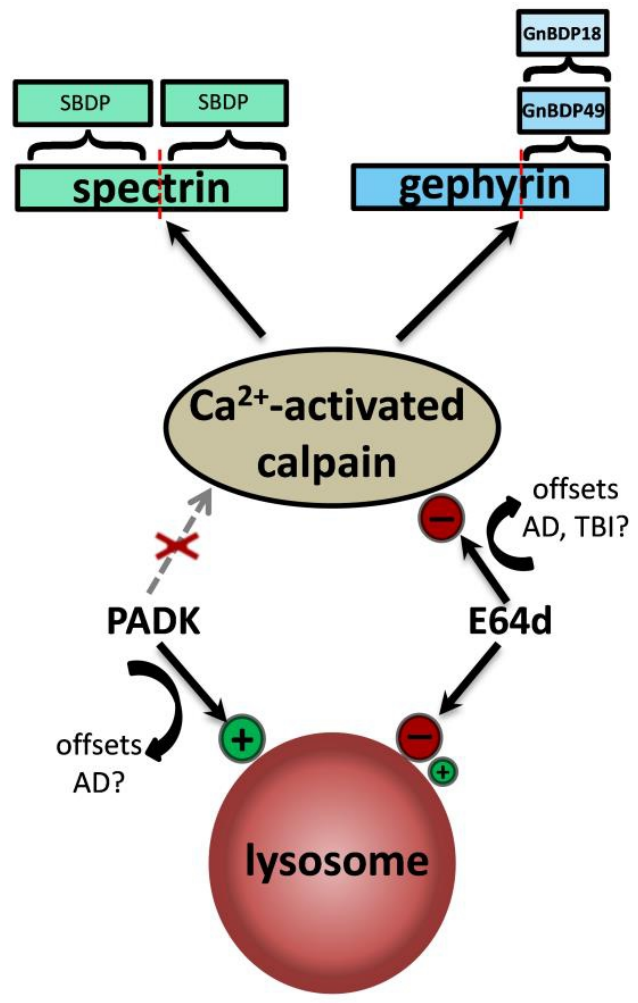

Figure 7. Differential pathways and therapeutic targets of PADK and E64d. PADK has a positive and selective effect on lysosomal CatB which likely explains its beneficial effect on AD-type protein accumulation pathology. E64d elicits low-level positive CatB modulation which may be part of its therapeutic action. In addition, evidence indicates that E64d potently blocks pathogenic, calpain-mediated cytoskeletal damage, and the compound is known to block a number of lysosomal enzymes. The singular action on calpain or the dual effect on calpain and the lysosomal pathway may explain E64d's protective results.

The results suggest that E64d targets the calcium-regulated enzyme calpain, an enzyme that is essential to a host of cellular processes and is known to participate in many pathologic cascades. E64d effectively reduced cytoskeletal degradation linked to neuropathology, evaluated by measuring spectrin and gephyrin proteolysis that was shown previously to be blocked by selective calpain inhibitors (Kawasaki et al., 1997; Vanderklish \& Bahr 2000). E64d and PADK exhibited disparate effects on the cytoskeletal damage in brain samples:

- $\quad$ E64d blocked calcium-mediated spectrin and gephyrin breakdown at $\geq 10 \mu \mathrm{M}$;

- $\quad$ E64d protected the parent gephyrin from calcium-mediated proteolysis at $\geq 10 \mu \mathrm{M}$;

- $\quad$ PADK did not block the induced cytoskeletal breakdown or protect the parent gephyrin. 
Of its different modulatory actions depicted in Figure 7, E64d's negative modulation of calpain alone very likely explains its protective effects against neurodegenerative insults, rather than through dual inhibition of both calpain and a family of lysosomal cathepsins (E64d is listed as an inhibitor of cathepsins B, F, H, K, and L). First, selective calpain inhibitors have been widely reported to protect against ischemic insults and TBI (see Vanderklish \& Bahr, 2000; Saatman et al., 2010; Ono et al., 2016). Second, E64d completely protected against the TBI-induced neuronal loss measured in CatB knockout mice (Hook et al., 2014b). Third, intracellular and hydrolyzed E64d, having a $0.04-\mu \mathrm{M} \mathrm{IC}_{50}$ for blocking calpain (Huang et al., 1992), is 350 times more potent towards calpain vs. CatB and has been shown to lower $\mathrm{A} \beta$ peptide levels and improve memory in APP transgenic mice (Hook et al., 2007, 2011, 2014a). In comparison, the calpain inhibitor BDA-410 is 748 times more potent towards calpain vs. CatB and exhibited similar memory improvement as well as restored normal synaptic functions in $\mathrm{AD}$ mice (Trinchese et al., 2008). Lastly, inhibition of cathepsins would not be a beneficial feature to add to a therapeutic for synaptic/cognitive protection since CatB-blocking levels of cysteine protease inhibitors were found to be toxic, causing the accumulation of APP fragments and reducing synaptic protein measures (Bahr et al., 1994). Similarly, E64d blocked lysosomal protein clearance leading to accumulating levels of the mutant huntingtin protein (Jeong et al., 2009).

Inhibiting calpain enzymes as a therapeutic avenue has long been a challenge since reducing calpain activity can $i$ ) negatively affect one of the many physiological roles for calpains and $i i)$ lead to adverse side effects. Even with these challenges, drug discovery efforts remain active to develop calpaintargeted strategies to treat cancer, cardiovascular diseases, drug abuse, and neurodegenerative disorders including latter stages of AD (see Ono et al., 2016; Liang et al., 2017).

Interestingly, the E64d neuroprotectant, shown to be effective in London-mutant APP mice, appears to be the first compound identified that is both a potent calpain inhibitor and a positive modulator of the lysosomal pathway through CatB up-regulation. As should be noted, a cautionary study reported that all APP-overexpressing mice exhibit a toxic protein accumulation that does not occur in AD brains and that calpain activation can be an artifact of APP overexpression (Saito et al., 2016). Notwithstanding, aberrant calpain activity may play a role in AD-related oxidative stress and age-related disruption of proteostasis that lead to lysosomal destabilization (see Nixon, 2000; Yamashima, 2016). Perhaps related, calpain inhibition has been suggested to promote protective protein clearance through inducing the autophagic-lysosomal pathway (Menzies et al., 2015; Watchon et al., 2017) and blocking the switch from $26 \mathrm{~S}$ to $20 \mathrm{~S}$ proteasomes in order to enhance 
regulated $26 \mathrm{~S}$ proteasomal protein degradation that is essential for neuronal health and survival (Huang et al., 2013). Thus, a potential therapeutic with calpain inhibitory and lysosomal enhancement properties may elicit two separate avenues for proteostasis protection. CatB enhancement, in fact, has been shown to protect against AD-type protein accumulation pathology and associated synaptic compromise in a chloroquine-induced model of protein accumulation stress (Bendiske \& Bahr, 2003; Butler et al., 2005; Rhyzikov \& Bahr, 2008) and in models directly treated with $A \beta_{42}$ (Park et al., 2016; Farizatto et al., 2017).

In summary, among the weak cysteine protease inhibitors assessed, the PADK compound was found to have a positive effect on CatB but did not appear to inhibit calpain. E64d, on the other hand, was demonstrated to be both a positive CatB modulator and a potent calpain inhibitor. This dual action suggests E64d has a unique ability to treat protein accumulation events related to AD.

\section{Acknowledgments}

This work was supported by the Coins for Alzheimer's Research Trust (BAB) and the NIH grant 5R25GM077634-04 to University of North Carolina - Pembroke. The funding agencies had no role in study design, data collection and analysis, or decision to publish. We thank Catherine M. Parisian for excellent proofreading and editing of this article and Ronald Long, Christopher Norton, Jessica Rice, and Yara Abumohsen for excellent technical assistance.

\section{References:}

1. Bahr, B.A. (2009). Lysosomal modulatory drugs for a broad strategy against protein accumulation disorders. Current Alzheimer Research, 6, 438-445.

2. Bahr, B.A., Abai, B., Gall, C., Vanderklish, P.W., Hoffman, K.B., \& Lynch, G. (1994). Induction of $\beta$ amyloid-containing polypeptides in hippocampus: evidence for a concomitant loss of synaptic proteins and interactions with an excitotoxin. Experimental Neurology, 129, 81-94.

3. Bahr, B.A., Wisniewski, M.L., \& Butler, D. (2012). Positive lysosomal modulation as a unique strategy to treat age-related protein accumulation diseases. Rejuvenation Research, 15, 189-197.

4. Bendiske, J. \& Bahr, B.A. (2003). Lysosomal activation is a compensatory response against protein accumulation and associated synaptopathogenesis-an approach for slowing Alzheimer disease? Journal of Neuropathology and Experimental Neurology, 62, 451-463.

5. Bendiske, J., Caba, E., Brown, Q.B., \& Bahr, B.A. (2002). Intracellular deposition, microtubule destabilization, and transport failure: an "early" 
pathogenic cascade leading to synaptic decline. Journal of Neuropathology and Experimental Neurology, 61, 640-650.

6. Burbulla, L.F., Song, P., Mazzulli, J.R., Zampese, E., Wong, Y.C., Jeon, S.,... Krainc, D. (2017). Dopamine oxidation mediates mitochondrial and lysosomal dysfunction in Parkinson's disease._Science, 357, 1255-1261.

7. Butler, D., Brown, Q.B., Chin, D.J., Batey, L., Karim, S., Mutneja, M.S., Karanian, D.A., \& Bahr, B.A. (2005). Cellular responses to protein accumulation involve autophagy and lysosomal enzyme activation. Rejuvenation Research, 8, 227-237.

8. Butler, D., Hwang, J., Estick, C., Nishiyama, A., Kumar, S.S., Baveghems, C.,... Bahr, B.A. (2011). Protective effects of positive lysosomal modulation in Alzheimer's disease transgenic mouse models. PLoS ONE, 6, e20501.

9. Cecarini, V., Bonfili, L., Cuccioloni, M., Mozzicafreddo, M., Rossi, G., Buizza, L.,... Eleuteri, A.M. (2012). Crosstalk between the ubiquitinproteasome system and autophagy in a human cellular model of Alzheimer's disease. Biochimica et Biophysica Acta, 1822, 1741-1751.

10. Cecarini, V., Bonfili, L., Cuccioloni, M., Mozzicafreddo, M., Rossi, G., Keller, J.N.,... Eleuteri, A.M. (2014). Wild type and mutant amyloid precursor proteins influence downstream effects of proteasome and autophagy inhibition. Biochimica et Biophysica Acta, 1842, 127-134.

11. Cermak, S., Kosicek, M., Mladenovic-Djordjevic, A., Smiljanic, K., Kanazir, S., \& Hecimovic, S. (2016). Loss of Cathepsin B and L Leads to Lysosomal Dysfunction, NPC-Like Cholesterol Sequestration and Accumulation of the Key Alzheimer's Proteins. PLoS ONE, 11, e0167428.

12. Ditaranto, K., Tekirian, T.L., \& Yang, A.J. (2001). Lysosomal membrane damage in soluble $\mathrm{A} \beta$-mediated cell death in Alzheimer's disease. Neurobiology of Disease, 8, 19-31.

13. Farizatto, K.L.G., Ikonne, U.S., Almeida, M.F., Ferrari, M.F.R., \& Bahr, B.A. (2017). Aß42-mediated proteasome inhibition and associated tau pathology in hippocampus are governed by a lysosomal response involving cathepsin B: Evidence for protective crosstalk between protein clearance pathways. PLOS ONE, 12, e0182895.

14. Fekete, C.D., Goz, R.U., Dinallo, S., Miralles, C.P., Chiou, T.T., Bear, J.,... De Blas, A.L. (2017). In vivo transgenic expression of collybistin in neurons of the rat cerebral cortex. Journal of Comparative Neurology, 525, 1291-1311.

15. Gavilán, E., Pintado, C., Gavilán, M.P., \& Daza, P., Sánchez-Aguayo, I., Castaño, A., \& Ruano, D. (2015). Age-related dysfunctions of the autophagy lysosomal pathway in hippocampal pyramidal neurons under proteasome stress._Neurobiology of Aging, 36, 1953-1963. 
16. Goldberg, A.L. (2003). Protein degradation and protection against misfolded or damaged proteins. Nature, 426, 895-899.

17. Hook, V., Kindy, M., \& Hook, G. (2007). Cysteine protease inhibitors effectively reduce in vivo levels of brain $\beta$-amyloid related to Alzheimer's disease. Biological Chemistry, 388, 247-252.

18. Hook, G., Hook, V., \& Kindy, M. (2011). The cysteine protease inhibitor, E64d, reduces brain Amyloid- $\beta$ and improves memory deficits in Alzheimer's disease animal models by inhibiting cathepsin B, but not BACE1, $\beta$-secretase activity. Journal of Alzheimer's Disease, 26, 387408.

19. Hook, G., Yu, J., Toneff, T., Kindy, M., \& Hook, V. (2014a). Brain pyroglutamate amyloid- $\beta$ is produced by cathepsin B and is reduced by the cysteine protease inhibitor E64d, representing a potential Alzheimer's disease therapeutic. Journal of Alzheimer's Disease, 41, 129-149.

20. Hook, G.R., Yu, J., Sipes, N., Pierschbacher, M.D., Hook, V., \& Kindy, M.S. (2014b).The cysteine protease cathepsin B is a key drug target and cysteine protease inhibitors are potential therapeutics for traumatic brain injury. Journal of Neurotrauma, 31, 515-529.

21. Huang, Z., McGowan, E.B., \& Detwiler, T.C. (1992). Ester and amide derivatives of E64c as inhibitors of platelet calpains. Journal of Medicinal Chemistry, 35, 2048-2054.

22. Huang, Q., Wang, H., Perry, S.W., \& Figueiredo-Pereira, M.E. (2013). Negative regulation of $26 \mathrm{~S}$ proteasome stability via calpain-mediated cleavage of Rpn10 subunit upon mitochondrial dysfunction in neurons. Journal of Biological Chemistry, 288, 12161-12174.

23. Inubushi, T., Kakegawa, H., Kishino, Y., \& Katunuma, N. (1994). Specific assay method for the activities of cathepsin L-type cysteine proteinases. Journal of Biochemistry, 116, 282-284.

24. Je Ma, C., Jung, W.J., Lee, K.Y., Kim, Y.C., \& Sung, S.H. (2009). Calpain inhibitory flavonoids isolated from Orostachys japonicus. Journal of Enzyme Inhibition and Medicinal Chemistry, 24, 676-679.

25. Jeon, K.H., Lee, E., Jun, K.Y., Eom, J.E., Kwak, S.Y., Na, Y.,... Kwon, Y. (2016). Neuroprotective effect of synthetic chalcone derivatives as competitive dual inhibitors against $\mu$-calpain and cathepsin $B$ through the downregulation of tau phosphorylation and insoluble $A \beta$ peptide formation. European Journal of Medicinal Chemistry, 121, 433-444.

26. Jeong, H., Then, F., Melia, T.J., Mazzulli, J.R., Cui, L., Savas, J.N.,... Krainc, D. (2009). Acetylation targets mutant huntingtin to autophagosomes for degradation. Cell, 137, 60-72.

27. Kawasaki, B.T., Hoffman, K.B., Yamamoto, R.S., \& Bahr, B.A. (1997). Variants of the receptor/channel clustering molecule gephyrin in brain: 
distinct distribution patterns, developmental profiles, and proteolytic cleavage by calpain. Journal of Neuroscience Research, 49, 381-388.

28. Liang, J., Li, J.L., Han, Y., Luo, Y.X., Xue, Y.X., Zhang, Y.,... Shi, J. (2017). Calpain-GRIP Signaling in Nucleus Accumbens Core Mediates the Reconsolidation of Drug Reward Memory. Journal of Neuroscience, 37, 8938-8951.

29. Mawuenyega, K.G., Sigurdson, W., Ovod, V., Munsell, L., Kasten, T., Morris, J.C.,... Bateman, R.J. (2010). Decreased clearance of CNS $\beta$ amyloid in Alzheimer's disease. Science, 330, 1774.

30. Mazzulli, J.R., Zunke, F., Tsunemi, T., Toker, N.J., Jeon, S., Burbulla, L.F.,... Krainc, D. (2016). Activation of $\beta$-glucocerebrosidase reduces pathological $\alpha$-synuclein and restores lysosomal function in Parkinson's Patient midbrain neurons. Journal of Neuroscience, 36, 7693-706.

31. Menzies, F.M., Garcia-Arencibia, M., Imarisio, S., O'Sullivan, N.C., Ricketts, T., Kent, B.A.,... Rubinsztein, D.C. (2015). Calpain inhibition mediates autophagy-dependent protection against polyglutamine toxicity. Cell Death and Differentiation, 22, 433-444.

32. Montagne, R., Baranzelli, A., Muharram, G., Catherine, L., Lesaffre, M., Vinchent, A.,... Tulasne, D. (2017). MET receptor variant R970C favors calpain-dependent generation of a fragment promoting epithelial cell scattering. Oncotarget, 8, 11268-11283.

33. Moon, H.Y., Becke, A., Berron, D., Becker, B., Sah, N., Benoni, G.,...van Praag, H. (2016). Running-induced systemic cathepsin b secretion is associated with memory function. Cell Metabolism, 24, 332-340.

34. Mueller-Steiner, S., Zhou,Y., Arai, H., Roberson, E.D., Sun, B., Chen, J.,... Gan, L. (2006). Antiamyloidogenic and neuroprotective functions of cathepsin B: implications for Alzheimer's disease. Neuron, 51, 703-714.

35. Nixon, R.A. (2000) A "protease activation cascade" in the pathogenesis of Alzheimer's disease. Annals of the New York Academy of Sciences, 924, 117-131.

36. Nixon, R.A. (2007). Autophagy, amyloidogenesis and Alzheimer disease. Journal of Cell Science, 120, 4081-4091.

37. Ono, Y., Saido, T.C., \& Sormachi, H. (2016). Calpain research and drug discovery: challenges and potential. Nature Reviews Drug Discovery, 15, 854-876.

38. Park, S.Y., Lee, H.R., Lee, W.S., Shin, H.K., Kim, H.Y., Hong, K.W., \& Kim, C.D. (2016). Cilostazol modulates autophagic degradation of $\beta$ amyloid peptide via SIRT1-coupled LKB1/AMPK $\alpha$ signaling in neuronal cells. PLoS ONE, 11, e0160620.

39. Pineda, J.A., Wang, K.K., \& Hayes, R.L. (2004). Biomarkers of proteolytic damage following traumatic brain injury. Brain Pathology, 14, 202-209. 
40. Ramalho, S.D., de Sousa, L.R., Burger, M.C., Lima, M.I., da Silva, M.F., Fernandes, J.B., Vieira, P.C. (2015). Evaluation of flavonols and derivatives as human cathepsin B inhibitor. Natural Product Research, 29, 2212-2214.

41. Rhyzikov, S. \& Bahr, B.A. (2008). Gephyrin alterations due to protein accumulation stress are reduced by the lysosomal modulator Z-Phe-Aladiazomethylketone. Journal of Molecular Neuroscience, 34,131-139.

42. Saatman, K.E., Creed, J., \& Raghupathi, R. (2010). Calpain as a therapeutic target in traumatic brain injury. Neurotherapeutics, 7, 31-42.

43. Saito, T., Matsuba, Y., Yamazaki, N., Hashimoto, S., \& Saido, T.C. (2016) Calpain activation in Alzheimer's model mice is an artifact of APP and presenilin overexpression. Journal of Neuroscience, 36, 9933-9936.

44. Sugiyama, K., Aida, T., Nomura, M., Takayanagi, R., Zeilhofer, H.U., \& Tanaka, K. (2017). Calpain-dependent degradation of nucleoporins contributes to motor neuron death in a mouse model of chronic excitotoxicity. Journal of Neuroscience, 37, 8830-8844.

45. Sun, B., Zhou, Y., Halabisky, B., Lo, I., Cho, S.H., Mueller-Steiner, S.,... Gan. L. (2008). Cystatin-C cathepsin B axis regulates amyloid $\beta$ levels and associated neuronal deficits in an animal model of Alzheimer's disease. Neuron, 60, 247-257.

46. Torres, M., Jimenez, S., Sanchez-Varo, R., Navarro, V., Trujillo-Estrada, L., Sanchez-Mejias, E.,...Vitorica, J. (2012). Defective lysosomal proteolysis and axonal transport are early pathogenic events that worsen with age leading to increased APP metabolism and synaptic $\mathrm{A} \beta$ in transgenic APP/PS1 hippocampus._Molecular Neurodegeneration, 7,59 .

47. Trinchese, F., Fá, M., Liu, S., Zhang, H., Hidalgo, A., Schmidt, S.D.,... Arancio, O. (2008). Inhibition of calpains improves memory and synaptic transmission in a mouse model of Alzheimer disease. Journal of Clinical Investigation, 118, 2796-2807.

48. Tsubokawa, T., Solaroglu, I., Yatsushige, H., Cahill, J., Yata, K., \& Zhang, J. (2006). Cathepsin and calpain inhibitor E64d attenuates matrix metalloproteinase-9 activity after focal cerebral ischemia in rats. Stroke, 37, 1888-1894.

49. Vanderklish, P.W. \& Bahr, B.A. (2000). The pathogenic activation of calpain: a marker and mediator of cellular toxicity and disease states. International Journal of Experimental Pathology, 81, 323-339.

50. Viswanathan, K., Hoover, D.J., Hwang, J., Wisniewski, M.L., Ikonne, U.S., Bahr, B.A., \& Wright, D. (2012). Nonpeptidic lysosomal modulators derived from Z-Phe-Aladiazomethylketone for treating protein accumulation diseases. ACS Medicinal Chemistry Letters, 3, 920-924. 
51. Wang, K.K.W. \& Yuen, P.W. (1994). Calpain inhibition: an overview of its therapeutic potential. Trends in Pharmacological Science, 15, 412-419.

52. Wang, C., Sun, B., Zhou, Y., Grubb, A., Gan, L. (2012). Cathepsin B degrades amyloid- $\beta$ in mice expressing wild-type human amyloid precursor protein. Journal of Biological Chemistry, 287, 39834-39841.

53. Wang, C., Telpoukhovskaia, M.A., Bahr, B.A., Chen, X., \& Gan, L. (2017). Endo-lysosomal dysfunction: a converging mechanism in neurodegenerative diseases. Current Opinion in Neurobiology, in press.

54. Watchon, M., Yuan, K. C., Mackovski, N., Svahn, A.J., Cole, N.J., Goldsbury, C.,... Laird, A.S. (2017). Calpain inhibition is protective in Machado-Joseph disease due to induction of autophagy. Journal of Neuroscience, 37, 7782-7794.

55. Weiss, E.S., Wang, K.K.W., Allen, J.G., Blue, M.E., Nwakanma, L.U., Liu, M.C.,... Baumgartner, W.A. (2009). aII-Spectrin breakdown products serve as novel markers of brain injury severity in a canine model of hypothermic circulatory arrest. Annals Thoracic Surgery, 88, 543-550.

56. Wu, J., Wang, Y., Kegel, K.B., Yoder, Y., Difiglia, M., Qin, Z., \& Lin, F. (2012). The regulation of N-terminal huntingtin (Htt552) acculumation by beclin 1. Acta Pharmalogica Sinica, 33, 743-751.

57. Yamashima, T. (2016). Can 'calpain-cathepsin hypothesis' explain Alzheimer neuronal death? Ageing Research Review, 32, 169-179.

58. Yang, D.S., Stavrides, P., Mohan, P.S., Kaushik, S., Kumar, A., Ohno, M.,... Nixon, R.A. (2011). Reversal of autophagy dysfunction in the TgCRND8 mouse model of Alzheimer's disease ameliorates amyloid pathologies and memory deficits. Brain, 134, 258-277. 